\title{
A 16-year prospective survey of Mycobacterium xenopi, Mycobacterium kansasii and Mycobacterium fortuitum infection in patients with HIV disease
}

Roberto Manfredi

From $16^{\text {th }}$ International Symposium on HIV and Emerging Infectious Diseases

Marseille, France. 24-26 March 2010

\section{Background}

A prompt and effective diagnosis and a timely treatment of atypical mycobacteriosis, and especially Mycobacterium kansasii, Mycobacterium xenopi, and Mycobacterium fortuitum disease, remains a serious challenge for clinicians engaged in the management of the immunocompromised host, including HIV disease.

\section{Methods}

Eighteen, twelve, and three HIV-infected patients with a microbiologically-confirmed M. kansasii, $M$. xenopi, and $M$. fortuitum respiratory infection respectively, have been observed in a 16-year period, out of over 4,700 hospitalizations performed because of HIV-associated disorders at our inpatient centre. These episodes were carefully evaluated from an epidemiological, bacteriological, clinical, and therapeutic point of view.

\section{Results}

In 15 out of the 33 overall episodes (45.5\%), a concurrent bacteremia was also retrieved, as a sign of disseminated infection. A rapid and significant reductin of the crude frequency of atypical mycobacteriosis as a major HIV-related complication, occurred shortly after the introduction of potent antiretroviral combinations (cART) in the year 1996. In fact, until early nineties, the lack of potent antiretroviral regimens made frequent the association of this opportunism with full-blown AIDS, a mean CD4+ lymphocyte count of around 20-50 cells/ $\mu \mathrm{L}$, and extremely variable chest $\mathrm{X}$-ray features and systemic presentations.
The recent detection of 9 further episodes of atypical mycobacteriosis in the year 2009 was due to a late recognition of a far advanced HIV disease (the so-called "AIDS presenters"), which were already complicated by multiple opportunistic disorders.

\section{Discussion}

M. kansasii, M. xenopi, and M. fortuitum respiratory and/or disseminated infection continues to occur, and pose relevant diagnostic problems, including late or missed identification due to slow culture and frequently concurrent opportunistic disease. Serious therapeutic difficulties, due to the unpredictable in vitro antimicrobial susceptibility profile of these organisms, and the need to start as soon as possible an effective combination therapy which should not interfere with other medications (especially cART), are also of concern.

Published: 11 May 2010

\section{doi:10.1186/1742-4690-7-S1-P36}

Cite this article as: Manfredi: A 16-year prospective survey of Mycobacterium xenopi, Mycobacterium kansasii and Mycobacterium fortuitum infection in patients with HIV disease. Retrovirology 2010 7(Suppl 1):P36. 